Revista Brasileira de Agricultura Irrigada v.8, nº. 2, p. 106 - 113, 2014

ISSN 1982-7679 (On-line)

Fortaleza, CE, INOVAGRI - http://www.inovagri.org.br

DOI: $10.7127 /$ rbai.v8n200236

Protocolo 236/14 - 01/12/2013 Aprovado em 28/03/2014

\title{
CARACTERIZAÇÃO DO ASPERSOR “MIDI” EM DIFERENTES ALTURAS DO PINO DISPERSOR
}

Jefferson Benigno Paula de Melo ${ }^{1}$, Gabriel Ferreira Cardoso Xavier ${ }^{2}$, Quemes Santos da Silva $^{3}$, Geuzimar Terração da Silva ${ }^{3}$, Marco Jorge Figueiras ${ }^{4}$, Leonardo Duarte Batista da Silva ${ }^{5}$

\section{RESUMO}

Objetivou-se por meio do presente estudo verificar o perfil de distribuição e o coeficiente de uniformidade em testes de laboratório para aspersores de impacto de média vazão, em duas posições distintas do pino dispersor. O experimento foi conduzido no Laboratório de Irrigação do Departamento de Engenharia da Universidade Federal Rural do Rio de Janeiro em Seropédica, RJ. Fora empregado o aspersor FABRIMAR MIDI 360, com a combinação de bocais 7,2 $\mathrm{mm}$ com bocal cego $(0 \mathrm{~mm})$ e $6,6 \mathrm{~mm}$ (bocal principal) com 3,6 $\mathrm{mm}$ (bocal secundário) na pressão de $30 \mathrm{mca}$ e com pino dispersor de 6,0 e 9,0 mm. As melhores médias de coeficiente de uniformidade foram obtidas empregando os bocais duplos com destaque para o com pino de $9 \mathrm{~mm}$, onde o mesmo é indicado até o espaçamento de 18x18 metros para sobreposição retangular e triangular. $\mathrm{Na}$ combinação 7,2x0 mm obteve-se desempenho satisfatório até o espaçamento de $15 \times 18 \mathrm{~m}$ na sobreposição retangular e 12x18 m na disposição triangular. $\mathrm{Na}$ analise do perfil de distribuição observou-se maiores volumes de água entre as distâncias de 0 a 5,5 m com decréscimo nas distâncias seguintes chegando a baixas precipitações nos dois metros finais no ensaio com bocais duplos. Com o bocal cego apenas o primeiro metro apresenta volume de água discrepante, mantendo valores próximos da média até 12 metros quando a precipitação se torna baixa. Os ensaios obtiveram raios efetivamente molhados de 16,5 metros para pino de $6 \mathrm{~mm}$ e com bocal cego e $16 \mathrm{~m}$ para pino de $9 \mathrm{~mm}$.

Palavras-chave: ensaio de distribuição, aspersores, análise da uniformidade.

\section{DESCRIPTION OF THE SPRINKLER "MIDI" IN DIFFERENT HEIGHTS PIN DISPERSER}

\footnotetext{
ABSTRACT

${ }^{1}$ Graduando em Engenharia Agrícola e Ambiental, bolsista de IT da FAPERJ, CEP 23890-000, Seropédica, RJ. Fone (21) 97903 6979. E-mail: jefferson-hexa@ hotmail.com

${ }^{2}$ Graduando em Engenharia Agrícola e Ambiental da UFRRJ, estagiário PROEXT/FAPERJ

${ }^{3}$ Graduando em Engenharia Agrícola e Ambiental da UFRRJ

${ }^{4}$ Engenheiro Agrícola, Mestre em Agricultura Orgânica, Doutorando em Ciência, Tecnologia e Inovação em Agropecuária da UFRRJ.

${ }^{5}$ Prof. Adjunto IV, Departamento de Engenharia. UFRRJ
} 
The present study aimed to verify the distribution profile and the coefficient of uniformity in laboratory tests to impact sprinklers medium flow. The experiment was conducted in the laboratory of Irrigation Engineering Department, Federal Rural University of Rio de Janeiro in Seropédica, RJ. Out MIDI FABRIMAR employed sprinkler 360, in combination with 7.2 $\mathrm{mm}$ nozzles with nozzle blind $(0 \mathrm{~mm}$ ) and $6.6 \mathrm{~mm}$ ( primary nozzle ) to $3.6 \mathrm{~mm}$ ( secondary nozzle ) pressure on the MCA and the pin 30 disperser 6 and $9 \mathrm{~mm}$. The best average uniformity coefficient were obtained using the double nozzles especially with the disperser pin $9 \mathrm{~mm}$, where the same are indicated by the spacing of $18 \times 18$ meters for rectangular and triangular overlap. In combination $\times 07.2 \mathrm{~mm}$ was obtained satisfactory rate until the spacing of $15 \times 18 \mathrm{~m}$ in overlapping rectangular and triangular layout in $12 \times 18 \mathrm{~m}$. In the analysis of the distribution profile was observed higher water volume between distances 0 to 5.5 myself a decrease in the following distances in low rainfall reaching 2 meters final for testing twin nozzles. With the blind nozzle meter displays only the first volume of differing water, maintaining good curve up to 12 meters when precipitation also becomes low.

Keywords: distribution test, sprinkler, uniformity analysis.

\section{INTRODUÇÃO}

Com a modernização da agricultura, vemse atingindo patamares de produção nunca alcançados. Isso é decorrente do maior conhecimento sobre as culturas e do emprego de novas tecnologias, dentro das quais se destaca o emprego da irrigação como um dos principais contribuintes para esses recordes de produção. PRADO e COLOMBO (2005) destacam que a proteção ambiental, a conservação de recursos hídricos e os custos de água e energia são características de extrema relevância para a agricultura e tem levado fabricantes de aspersores e pesquisadores em geral a desenvolver produtos mais eficientes, respeitando essas características.

Uma forma de atingir esses objetivos é proporcionar uma maior uniformidade na distribuição de água no processo de irrigação. $\mathrm{Na}$ irrigação por aspersão a aplicação eficiente de água está diretamente ligada à uniformidade desta, ou seja, baixos valores de coeficientes de uniformidade levam a uma má distribuição da água no solo. A desuniformidade de distribuição de água dos sistemas de irrigação acarretando superirrigação em parte da área, eleva os custos de produção, podem acarretar, ainda outros problemas ao meio ambiente, como contaminação de águas subterrâneas, devido à percolação profunda, promovidos pela lixiviação de nutrientes, afirma Capra e Scicolone (2004) citado por Martins et al. (2012).

A uniformidade de distribuição de água só é garantida quando são avaliadas a características dos equipamentos empregados, especialmente dos aspersores. Os resultados obtidos servirão como base para dimensionamento de projetos de irrigação por aspersão. Bernardo et al. (2008) ressaltam que para promover o desenvolvimento de pesquisas e de novos aspersores, ter um melhor controle da qualidade de fabricação e 
utilização dos aspersores por parte do usuário é necessário à obtenção precisa do perfil de distribuição.

KELLER e BLIESNER (1990), Bernardo (2005), destacam que o perfil de distribuição e o coeficiente de uniformidade são característicos do modelo de aspersor e, em geral, dependem das características físicas, das condições operacionais e dos intemperes proporcionados pelo clima.

Segundo Rocha et al. (1999) muitos modelos de coeficientes de uniformidade foram desenvolvidos a partir do coeficiente de uniformidade de Christiansen (CUC) (1942), que utiliza o desvio médio absoluto como medida de dispersão e adota como bons índices de uniformidade, $80 \%$ como valor mínimo aceitável. Frizzone (1992) citado por Rocha et al. (1999) ressalta que nenhum dos demais coeficientes substitui com vantagem o CUC. Pela sua facilidade de operação e sua simplicidade CUC é o método mais conhecido e empregado para análise de coeficientes de uniformidade.

Com o intuito de facilitar o desenvolvimento de pesquisas e avaliação dos sistemas de irrigação, a Associação Brasileira de Normas Técnicas (ABNT), lançou em janeiro de 2000, a NBR 7749-1, 2000. Que contem normas e métodos para ensaios de uniformidade e dispersão.

Com isso a ABNT (2000), proporcionou a melhoria dos equipamentos de aspersão, uma vez que passou a ser facilitada e mais frequente a obtenção de dados característicos de cada aspersor. Com o intuito de saber de que forma era distribuída a lâmina de água no solo surgiu o interesse de caracterizar o perfil de distribuição e o coeficiente de uniformidade em testes de laboratório para aspersor de impacto de média vazão.

Objetivou-se por meio do presente trabalho verificar o perfil de distribuição e o coeficiente de uniformidade em testes de laboratório para aspersores de impacto de média vazão, em duas distintas posições do pino dispersor.

\section{MATERIAIS E MÉTODOS}

O presente estudo foi conduzido no Laboratório de Hidráulica e Irrigação pertencente ao Departamento de Engenharia da Universidade Federal Rural do Rio de Janeiro (UFRRJ), no município de Seropédica - RJ. Os trabalhos foram realizados empregando-se dois aspersores de impacto de média vazão, FABRIMAR MIDI 360, os quais apresentaram as combinações de bocais $7,2 \mathrm{~mm}$ com bocal cego (0 mm) e 6,6 mm (bocal principal) com 3,6 $\mathrm{mm}$ (bocal secundário) com pino dispersor com medidas de 6 e $9 \mathrm{~mm}$, trabalhando com uma pressão de serviço de 30 mca.

Trabalhando de acordo com as especificações da ABNT (2000), os ensaios foram conduzidos em casa de vegetação, a fim de evitar interferências proporcionadas por intemperes climáticos, (em destaque o vento). Foram utilizados 40 coletores plásticos, dispostos em linha com espaçamentos de $0,5 \mathrm{~m}$ entre eles. Nos teste foram utilizadas aberturas frontais de 0,7 m. Segundo Silva et al. (2012) aberturas frontais, menores que $0,6 \mathrm{~m}$, interferem significativamente no volume de água 
encontrado nos coletores na distância de até três metros, mascarando assim o foco do presente trabalho, que é a análise do perfil de distribuição, desta forma alterando também os valores do coeficiente de uniformidade.

Para os testes o fluxo de água, após passar por um filtro de tela (150 mesh), foi conduzido por uma tubulação de PVC de 1 polegada $(0,0254 \mathrm{~m})$ até a campânula construída em alvenaria onde o aspersor estava instalado. $\mathrm{O}$ recalque da água foi feito por meio de um conjunto moto bomba com potência de $5 \mathrm{cv}$.

Após uma hora de funcionamento do conjunto moto bomba/aspersor foram realizadas as leituras dos volumes de água, fazendo uso de uma proveta volumétrica graduada com $0,1 \mathrm{~mm}$ de precisão, em cada uma das três repetições com os dois modelos de aspersores de impacto de média vazão, sendo um dos modelos com combinação de bocais $6,6 \times 3,6 \mathrm{~mm}$ com pino dispersor de 6 e $9 \mathrm{~mm}$ e o outro 7,2x0 $\mathrm{mm}$. Os volumes foram obtidos realizando a média aritmética dos volumes coletados em três repetições em cada teste.

Objetivando validar os testes, admitindo-se um erro máximo de $5 \%$, foi realizado o procedimento para a obtenção da vazão do aspersor, realizou-se a comparação dos resultados obtidos em um medidor de vazão eletromagnético digital modelo: Optiflux 2000, fabricante Conaut, com os valores encontrados nos coletores. As leituras feitas dos perfis de distribuição, foram calculadas com o auxílio do software EnsAsper versão 0.9.50., dando origem ao raio efetivo molhado e a uniformidade de distribuição. A avaliação dos perfis de distribuição foram todas realizadas em planilhas eletrônicas, originadas pelo mesmo programa.

\section{RESULTADO E DISCUSSÃO}

Como pode ser observado na Tabela 1 as melhores médias de coeficientes de uniformidade de distribuição foram encontrados nos testes com bocais duplos na combinação 6,6x3,6 mm com pino dispersor de $9 \mathrm{~mm}$. Estes atingiram os melhores coeficientes de uniformidade em todos os espaçamentos se comparado com os demais aspersores testados. E seus bons valores de CUC foram observados até o espaçamento de $18 \times 18 \mathrm{~m}$ tanto para a sobreposição retangular quanto para a triangular. Índices semelhantes foram encontrados no teste com pino dispersor de $6 \mathrm{~mm}$, também sendo indicados para a distância acima citada, apresentando raio efetivo molhado de $16 \mathrm{~m}$ no primeiro caso e $16,5 \mathrm{~m}$ no segundo, expressando índices bem acima do indicado como aceitável para este tipo de teste.

Tabela 1: Coeficiente de Uniformidade Christiansen (CUC) (\%) das sobreposições retangulares de triangulares.

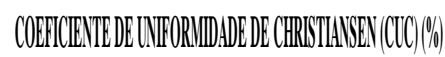

\begin{tabular}{|c|c|c|c|c|c|c|}
\hline \multirow{3}{*}{ BPPACANATIO(m) } & \multicolumn{2}{|c|}{ 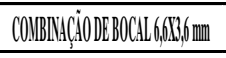 } & \multicolumn{2}{|c|}{ 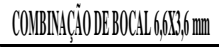 } & \multicolumn{2}{|c|}{ 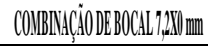 } \\
\hline & \multicolumn{2}{|c|}{ PINODSPERRSOR $6 \mathrm{~mm}$} & \multicolumn{2}{|c|}{ PINODISPERSOR9M } & \multicolumn{2}{|c|}{ PNODSPPERSOROnm } \\
\hline & REEANGULAR & TRHAGGLLR & RETANGLLAR & TRAHGULLR & REEAHGLLAR & TRHAGULAR \\
\hline 686 & 96, 19 & 99,87 & 9,, & 96.28 & 9,993 & $9,1,4$ \\
\hline $6 X 12$ & $9,6,63$ & $94,1.1$ & 97,9 & 96,55 & $9,4,5$ & 9,39 \\
\hline 12112 & $9,9,4$ & 99.1.8. & 96.217 & 94,97 & 86,2 & 91,85 \\
\hline $\mid 2115$ & $91,1,3$ & 8927 & 9.61 & $9 /, 65$ & 890 & 82,6 \\
\hline 1218 & 8999 & 92,8 & 92,25 & 0953 & 87,9 & 86,68 \\
\hline $15 \times 15$ & 87,97 & $9,1,19$ & 8899 & 99000 & 8,1,1 & 79,93 \\
\hline $15 \times 18$ & 801,18 & 80,2 & 89,9 & $9.6,5$ & 80,5 & 76,0 \\
\hline 1818 & 91,8 & 80,61 & 91,53 & $9,0,40$ & 88,18 & $7,5,1$ \\
\hline 1824 & 74,0 & $74,0^{5}$ & 13:00 & 1282 & 7287 & $24+10$ \\
\hline 2424 & 6,58 & $71, B$ & 60,12 & 6821 & 09,38 & $6,0,8$ \\
\hline RAIOEEETTIOYOOHHOO(m) & 1 & & th & & 16 & in \\
\hline
\end{tabular}


Já o teste com bocal cego na combinação 7,2 × $0 \mathrm{~mm}$ sem pino dispersor, apresentaram boas médias de coeficiente de uniformidade acima dos índices indicados para esse tipo de teste até o espaçamento de $15 \times 18 \mathrm{~m}$ para a organização retangular e 12 x $18 \mathrm{~m}$ para a sobreposição triangular, apresentando raio efetivo molhado de $16,50 \mathrm{~m}$.

Como pode ser observado na Figura 1a, o teste de aspersão de bocais duplos com pino dispersor de $9 \mathrm{~mm}$ apresentou resultados muito superiores ao valor mínimo satisfatório no perfil de distribuição, apresentando um maior volume de água no intervalo de 0 a 5,5 $\mathrm{m}$ com a maior lâmina de água sendo atingida entre as distâncias 1,5 a $5,5 \mathrm{~m}$ e um decréscimo constante nas distâncias seguintes, contribuindo assim para os excelentes resultados de uniformidade encontrados no CUC de 93,23\% observados na Figura 1b, que representa a sobreposição retangular com espaçamento $12 \times 18 \mathrm{~m}$. Entretanto nos dois metros finais o volume de distribuição de água atinge baixos valores de precipitação.

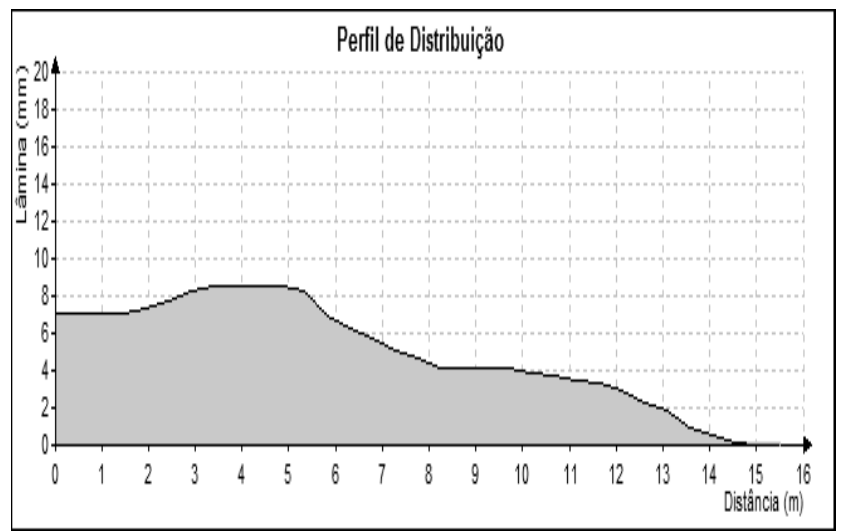

Figura 1a: Perfil de Distribuição de bocais 6,6 x 3,6 $\mathrm{m}$ com pino dispersor de $9 \mathrm{~mm}$

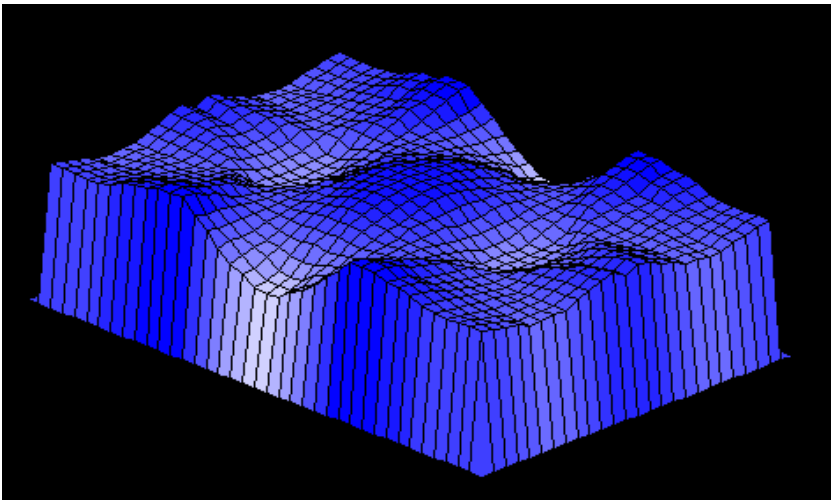

Figura 1b: Sobreposição retangular da área.

A partir dos dados que confeccionaram a Figura 1a foi gerada a superfície representada na Figura $1 b$, que indica a sobreposição de quatro aspersores alocados no espaçamento anteriormente citado. Nota-se dois picos de precipitação: o primeiro pode ser visualizado no perfil de distribuição; e o segundo, localizado no centro da figura, indica o ponto da extremidade do raio efetivo molhado, onde se tem a precipitação proveniente da intersecção dos quatros aspersores usados no teste pelo software EnsAsper.

Já o perfil de distribuição dos testes de bocal duplo com pino dispersor de $6 \mathrm{~mm}$ apresentou-se com uma inconsistência maior dos valores médios coletados ao longo do perfil se comparado com o primeiro aspersor citado, sendo encontrado o maior volume de água no primeiro metro e com um decréscimo inconstante e maiores volumes de água até a distância de $5 \mathrm{~m}$, quando o mesmo passa a reduzir de forma constante os valores encontrados até a distância de 14 metros, atingindo valores baixos de precipitação, com lâmina de água inferior a $2 \mathrm{~mm}$. 


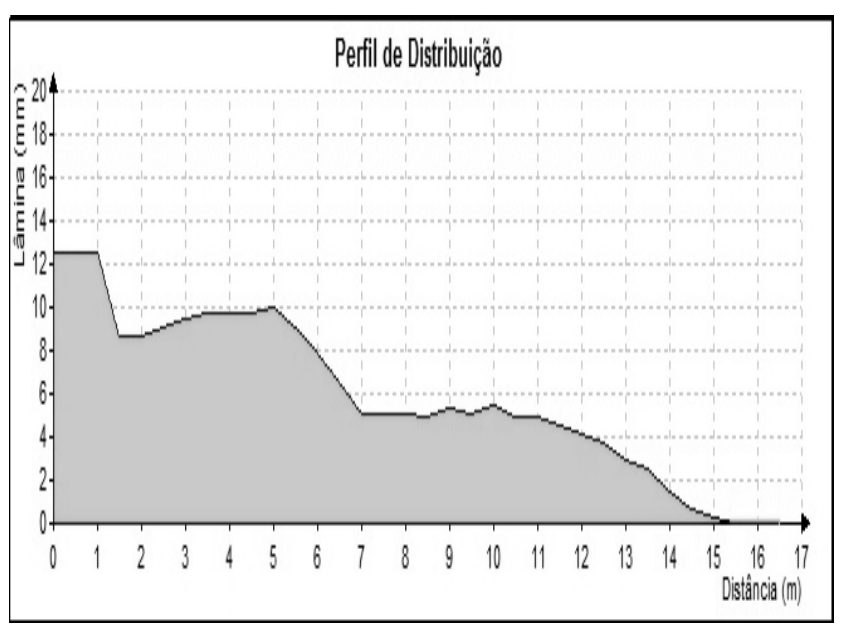

Figura 2a: Perfil de Distribuição de bocais duplos com pino dispersor de $6 \mathrm{~mm}$

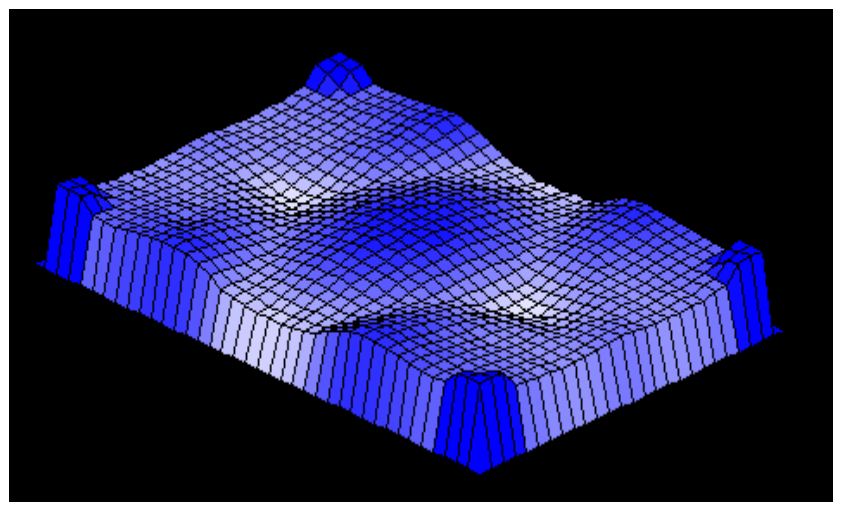

Figura 2b: Sobreposição retangular da área.

Os valores do coeficiente de uniformidade se assemelharam aos encontrados nos teste com pino dispersor de $9 \mathrm{~mm}$, com CUC chegando a $89,97 \%$ para o espaçamento $12 \times 18 \mathrm{~m}$ na sobreposição retangular de quatro aspersores apresentados na Figura 2b. Nesta pode-se observar três picos de precipitação, onde os dois primeiros são visualizados no perfil e o terceiro no centro da imagem gerada pelo programa acima citado. Na Figura $2 b$ pode-se observar dois picos de maior volume de água: um localizado no primeiro metro e o segundo a aproximadamente $5 \mathrm{~m}$. O terceiro, no centro da imagem gerada pelo programa, que mostra a intersecção dos raios molhados de cada aspersor.

Observando o perfil de distribuição do aspersor com bocal cego apresentado na Figura 3a, nos testes, o mesmo apresentou um grande volume de água no primeiro metro de distribuição, havendo um decréscimo constante e com precipitações satisfatórias até a distância de $12 \mathrm{~m}$. Desta forma o mesmo apresentou bons valores de coeficiente de uniformidade CUC de $87,79 \%$ na sobreposição retangular com espaçamento de 12x18 m observados na Figura 3b, chegando a índices aceitáveis CUC acima de $80 \%$ até o espaçamento $15 \times 18 \mathrm{~m}$.

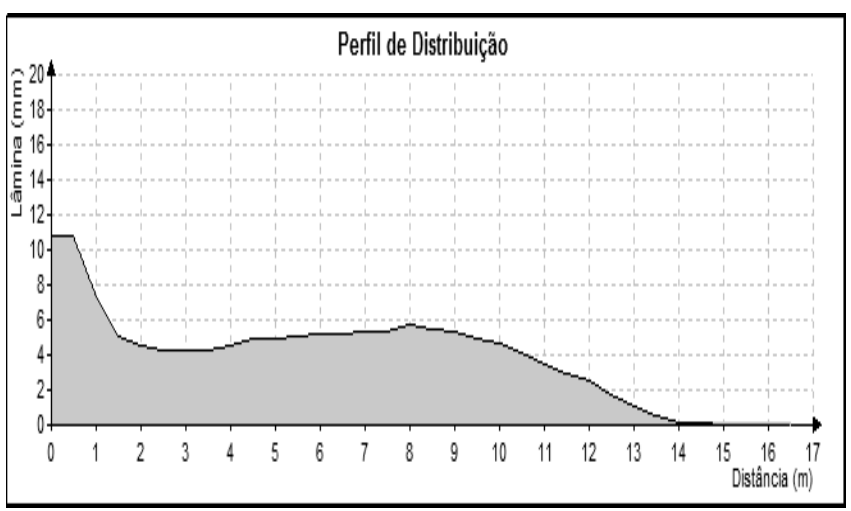

Figura 3a: Perfil de Distribuição de bocal 7,2 mm com bocal cego.

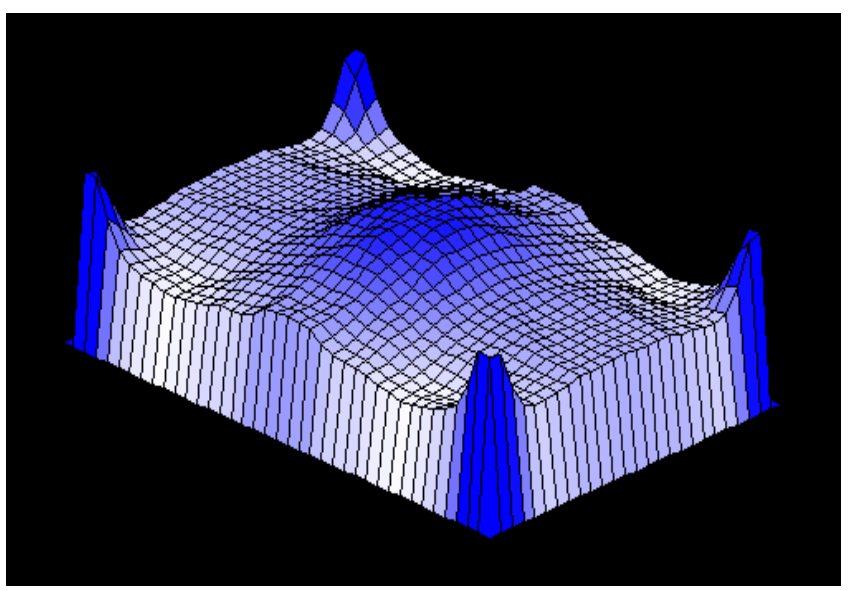

Figura 3b: Sobreposição retangular da área. 


\section{CONCLUSÃO}

Diante do presente estudo, pode-se concluir que todos os aspersores apresentaram índices satisfatórios de coeficiente de uniformidade. Sendo que os com bocais duplos com ambas as alturas do pino dispersor, apresentaram coeficientes de uniformidade mais satisfatórios até as distâncias de $18 \times 18 \mathrm{~m}$. Com destaque para o aspersor que apresentava pino dispersor de $9 \mathrm{~mm}$, que de todos os aspersores testados foi quem apresentou os valores mais expressivos, só que com menor raio efetivo molhado.

Já o aspersor com bocal cego foi quem apresentou os menores valores de coeficientes de uniformidade, mesmo atingindo uma maior distância de raio efetivo molhado juntamente com o aspersor de bocal duplo com altura de pino dispersor de $6 \mathrm{~mm}$, só que sua falta de uniformidade podem fazer com que superirrigue uma determinada área, levando a um aumento no custo de produção, sendo o mesmo indicado para áreas limites de $15 \times 18 \mathrm{~m}$ para organização retangular e $12 \times 18 \mathrm{~m}$ para a disposição triangular dos aspersores.

Conclui-se também que o aspersor que poderá proporcionar o menor custo de produção quando empregado é o com altura de pino dispersor de $9 \mathrm{~mm}$, pois sua uniformidade de distribuição de água leva á uma quantidade mais precisa de lâmina de água que cai a cada metro, atendendo melhor a necessidade de cada planta, diminuindo as perdas de nutrientes e evitando a contaminação do lençol freático ocorridos pela lixiviação de nutrientes.

\section{AGRADECIMENTOS}

A FAPERJ pela concessão da bolsa de estágio, a Universidade Federal Rural do Rio de Janeiro por conceder o espaço físico para a realização dos testes e a empresa FABRIMAR S.A. por disponibilizar os materiais e equipamentos utilizados nos testes.

\section{REFERENCIAS BIBLIOGRÁFICAS}

ABNT - Associação Brasileira de Normas Técnicas. Equipamentos de irrigação agrícola aspersores rotativos; Parte 2: uniformidade de distribuição e métodos de ensaio. Janeiro 2000, $6 \mathrm{p}$.

BERNARDO, S. , SOARES A. A., MANTOVANI, E. C. Manual de irrigação. Imprensa Universitária. Ed. 8. Viçosa, 300p. 2008.

CHISTIANSEN, J. E. Irrigation by Sprinkling. Berkely: California Agricultural Station. Bulletin 670, 124p, 1942.

KELLER, J.; BLIESNER, R.D. Sprinkle and trickle irrigation. New York: van Nostrand Reinhold, 615p. 1990.

MARTINS, P. E. S.; ZANINI, J. R.; SILVA, E. R.; LEMOS FILHO, M. A. F. Perfil radial e uniformidade de precipitação do aspersor NaanDanjain 427, em função da regulagem do defletor. Revista Brasileira de Engenharia Agrícola e Ambiental, v.16, n.1, p. 3-9, 2012.

PRADO, G. do; COLOMBO, A. Caracterização técnica do aspersor Plona - RL 300. Irriga, Botucatu, v.10, n.1, p. 53-63, janeiro-abril, 2005.

ROCHA, E. M. M.; COSTA, R. N. T.; MAPURUNGA, S. M. S.; CASTRO, P. T. Uniformidade de distribuição de água por 
aspersão convencional na superfície e no perfil do solo. Revista Brasileira de Engenharia Agrícola e Ambiental, v.3, n.2, p. 154-160, 1999.

SILVA, Q. S.; GUIMARÃES, G. P.; MORETTI, M. A. R.; FRANCISCO, J. P.; JORGE, M. J.;
SILVA, L. D. B. Análise do perfil de distribuição de água em teste de aspersão feitos em laboratório com diferentes aberturas frontais. In: INOVAGRI INTERNATIONAL MEETING \& IV WORKSHOP INTERNACIONAL DE INOVAÇÃO TECNOLÓGICA NA IRRIGAÇÃO, 05., 2012, Fortaleza. Resumos... Fortaleza. 